\title{
Stability of Radiomic Features in Fast Scanned FLAIR for Acute Ischemic Stroke Patients: A Comparison With Conventional FLAIR
}

Leehi Joo

Department of Radiology and Research Institute of Radiology, Asan Medical Center, University of Ulsan College of Medicine

Seung Chai Jung ( $\nabla$ dynamics79@gmail.com)

Department of Radiology and Research Institute of Radiology, Asan Medical Center, University of Ulsan College of Medicine

Hyunna Lee

Bigdata Research Center, Asan Institute for Life Science, Asan Medical Center

\section{Seo Young Park}

Department of Clinical Epidemiology and Biostatistics, Asan Medical Center, University of Ulsan College of Medicine

\section{Minjae Kim}

Department of Radiology and Research Institute of Radiology, Asan Medical Center, University of Ulsan College of Medicine

\section{Ji Eun Park}

Department of Radiology and Research Institute of Radiology, Asan Medical Center, University of Ulsan College of Medicine

\section{Keum Mi Choi}

Department of Radiology and Research Institute of Radiology, Asan Medical Center, University of Ulsan College of Medicine

\section{Research Article}

Keywords: echo-planar imaging (EPI), repetition time (TR)-FLAIR, radiomics, stability and robustness

Posted Date: March 2nd, 2021

DOl: https://doi.org/10.21203/rs.3.rs-243918/v1

License: () (1) This work is licensed under a Creative Commons Attribution 4.0 International License. Read Full License 
Version of Record: A version of this preprint was published at Scientific Reports on August 25th, 2021. See the published version at https://doi.org/10.1038/s41598-021-96621-z. 


\section{Abstract}

From May 2015 to June 2016, 296 patients undergoing 1.5-Tesla MRI for symptoms of acute ischemic stroke were retrospectively enrolled. Conventional, echo-planar imaging (EPI) and echo train length (ETL)FLAIR were simultaneously obtained in 118 patients (1st group), and conventional, ETL-, and repetition time (TR)-FLAIR were simultaneously obtained in 178 patients (2nd group). A total of 595 radiomics features were extracted from one region-of-interest (ROI) reflecting the acute and chronic ischemic hyperintensity, and concordance correlation coefficients (CCC) of the radiomics features were calculated between the fast scanned and conventional FLAIR for paired patients (1st group and 2nd group). Stabilities of the radiomics features were compared with the proportions of features with a CCC higher than 0.85 , which were considered to be stable in the fast scanned FLAIR. EPI-FLAIR showed higher proportions of stable features than ETL-FLAIR, and TR-FLAIR also showed higher proportions of stable features than ETL-FLAIR, both in acute and chronic ischemic hyperintensities of whole- and intersection masks $(p<.002)$. Radiomics features in fast scanned FLAIR showed variable stabilities according to the sequences compared with conventional FLAIR. Therefore, radiomics features may be used cautiously in applications for feature analysis as their stability and robustness can be variable.

\section{Introduction}

The paradigm is shifting from qualitative visual assessment of medical imaging to quantitative data analysis with the development of high-throughput mining of low- to high dimensional data. Radiomic features are considered to be an important alternative for interpretation and analysis of medical images and to predict lesion characteristics with numerous features, from first-order to high-order features ${ }^{1-4}$.

However, radiomic features can have limitations in their reproducibility or stability. The stability of radiomic features is still challenging with a lack of standardization during image acquisition, reconstruction, segmentation and analyses even though standardized image processing and feature computation have allowed radiomic features to be stable ${ }^{4}$. Among the various types of medical imaging, magnetic resonance imaging (MRI) has a variety of imaging acquisition methods and combinations of complicated parameters even in the same imaging sequences, which makes it difficult to apply radiomic features to MRI.

Fast scanned techniques are essential in the acquisition of MRI because of the major limitation of MRI, the need for a long scan time, particularly in emergency situations such as after a suspected cerebral acute ischemic stroke ${ }^{5}$. Fast scanned images have been realized by using conventional methods such as echo-planar imaging, parallel imaging, echo train length and recently introduced advanced techniques

such as compressed sensing and simultaneous multi-slice acquisition, and so on ${ }^{6-13}$. The various techniques have resulted in a very complicated combination of imaging parameters, which can hamper the acquisition of stable radiomic features. 
Fluid attenuated inversion recovery (FLAIR) is very commonly used and essential sequence for the evaluation of cerebral acute ischemic stroke patients ${ }^{9-11,14-17}$. Therefore, FLAIR is an important candidate for the application of radiomic features. However, there have been attempts to reduce the scan time of FLAIR for a long time, which resulted in various parameters of FLAIR. The conventional methods

are echo-planar imaging, parallel imaging, and echo train length ${ }^{6,9-12}$. Nevertheless, the stability of the radiomic features have been poorly investigated.

We hypothesized that radiomic features from fast scanned FLAIR show variability relative to conventional FLAIR.

Therefore, the aim of our study was to investigate the stability of radiomic features from various fast scanned FLAIR images in patients with acute ischemic stroke, and to compare the agreement of the radiomic features with conventional FLAIR as a reference standard.

\section{Results}

\section{Stability of radiomic features in fast scanned FLAIR compared with conventional FLAIR}

The proportions of stable radiomic features in the first group were 13.8\% (82/595) in EPI-FLAIR and 5.5\% (33/595) in ETL-FLAIR for acute ischemic hyperintensity and 15.6\% (93/595) in EPI-FLAIR and 5.1\% $(30 / 595)$ in ETL-FLAIR for chronic ischemic hyperintensity. Proportions of stable radiomic features in the second group were 16.8\% (100/595) in TR-FLAIR and 1.8\% (11/595) in ETL-FLAIR for acute ischemic hyperintensity and 13.0\% (77/595) in TR-FLAIR and 0.7\% (4/595) in ETL-FLAIR for chronic ischemic hyperintensity. EPI-FLAIR and TR-FLAIR showed significantly higher proportions of stable radiomic features than those of ETL-FLAIR $(p<.001)$. Proportions of stable radiomic features in the first group were 9.7\% (58/595) in the EPI-FLAIR intersection and 3.4\% (20/595) in the ETL-FLAIR intersection for acute ischemic hyperintensity and $18.0 \%$ (107/595) in the EPI-FLAIR intersection and 11.6\% (69/595) in the ETL-FLAIR intersection for chronic ischemic hyperintensity. Proportions of stable radiomic features in the second group were 12.1\% (72/595) in the TR-FLAIR intersection and 3.9\% (23/595) in the ETL-FLAIR intersection for acute ischemic hyperintensity and 9.6\% (57/595) in the TR-FLAIR intersection and 1.2\% (7/595) in the ETL-FLAIR intersection for chronic ischemic hyperintensity. EPI-FLAIR and TR-FLAIR showed significantly higher proportions of stable radiomic features than those of ETL-FLAIR $(p<.002)$. The detailed results are listed in Table 1. 
Table 1

Reproducibility of radiomics features in fast scanned FLAIR compared with original FLAIR as a reference standard

\section{Whole}

First group

EPI
ETL
Second group

TR

ETL

Proportions of stable radiomics features (CCC $>.85)$

\begin{tabular}{|c|c|c|c|c|c|c|}
\hline AlH & $\begin{array}{l}13.8 \% \\
(82 / 595)\end{array}$ & $\begin{array}{l}5.5 \% \\
(33 / 595)\end{array}$ & $<.001$ & $\begin{array}{l}16.8 \% \\
(100 / 595)\end{array}$ & $\begin{array}{l}1.8 \% \\
(11 / 595)\end{array}$ & $<.001$ \\
\hline $\mathrm{ClH}$ & $\begin{array}{l}15.6 \% \\
(93 / 595)\end{array}$ & $\begin{array}{l}5.0 \% \\
(30 / 595)\end{array}$ & $<.001$ & $\begin{array}{l}12.9 \% \\
(77 / 595)\end{array}$ & $\begin{array}{l}0.7 \% \\
(4 / 595)\end{array}$ & $<.001$ \\
\hline Overall & $\begin{array}{l}16.0 \% \\
(95 / 595)\end{array}$ & $\begin{array}{l}8.1 \% \\
(48 / 595)\end{array}$ & $<.001$ & $\begin{array}{l}22.9 \% \\
(136 / 595)\end{array}$ & $\begin{array}{l}2.0 \% \\
(12 / 595)\end{array}$ & $<.001$ \\
\hline
\end{tabular}

Median CCC

\begin{tabular}{|c|c|c|c|c|}
\hline $\begin{array}{l}0.60(0.13- \\
0.80)\end{array}$ & $\begin{array}{l}0.58(0.23- \\
0.75)\end{array}$ & .557 & $\begin{array}{l}0.66(0.32- \\
0.81)\end{array}$ & $\begin{array}{l}0.43 \\
(0.16- \\
0.66)\end{array}$ \\
\hline
\end{tabular}

$\mathrm{ClH}$

$0.37(0.06-$

$0.76)$

$0.47(0.16-\quad .011$

$0.68)$

$0.44(0.23-$

$0.67)$

0.30

(0.12$<.001$

$0.45)$

Overall

$0.62(0.14-$
$0.82)$

$0.57(0.22-\quad .607$

$0.76)$

$0.68(0.32-$
$0.84)$

0.47

(0.18-

0.67 )

Intersection First group

Second group

EPI

ETL

TR

ETL

$\mathbf{P}$

Proportions of stable radiomics features (CCC

$>.85)$

\begin{tabular}{lllllll} 
AlH & $9.7 \%$ & $3.4 \%$ & $<$ & $12.1 \%$ & $3.9 \%$ & $<$ \\
& $(58 / 595)$ & $(20 / 595)$ & 0.001 & $(72 / 595)$ & $(23 / 595)$ & 0.001 \\
CIH & $18.0 \%$ & $11.6 \%$ & 0.002 & $9.6 \%$ & $1.2 \%$ & $<$ \\
& $(107 / 595)$ & $(69 / 595)$ & & $(57 / 595)$ & $(7 / 595)$ & 0.001 \\
\multirow{2}{*}{ Overall } & $8.7 \%$ & $2.5 \%$ & $<$ & $13.3 \%$ & $3.4 \%$ & $<$ \\
& $(52 / 595)$ & $(15 / 595)$ & 0.001 & $(79 / 595)$ & $(20 / 595)$ & 0.001
\end{tabular}

* EPI $=$ echo-planar imaging, $E T L=$ echo train length, $T R=$ repetition time,$P=p$ value,$C C C=$ concordance correlation coefficients, AlH = acute ischemic hyperintensity, $\mathrm{ClH}=$ chronic ischemic hyperintensity

** Parentheses indicate numbers of radiomics features in proportions of stable radiomics features or indicate $95 \%$ confidence intervals. 


\begin{tabular}{|lllllll}
\hline Whole & \multicolumn{2}{l}{ First group } & & \multicolumn{3}{l}{ Second group } \\
\cline { 2 - 7 } & EPI & ETL & P & TR & ETL & P \\
\hline Median CCC & & & & & \\
AlH & $0.45(0.10-$ & 0.46 & $<$ & $0.56(0.27-$ & 0.52 & $<$ \\
& $0.69)$ & $(0.15-$ & 0.001 & $0.73)$ & $(0.25-$ & 0.001 \\
& & $0.64)$ & & & $0.69)$ & \\
CIH & $0.41(0.09-$ & $0.55(0.28-$ & $<$ & $0.46(0.24-$ & 0.32 & $<$ \\
& $0.75)$ & $0.73)$ & 0.001 & $0.63)$ & $(0.12-$ & 0.001 \\
& & & & & $0.48)$ & \\
\hline Overall & $0.49(0.13-$ & $0.52(0.21-$ & 0.348 & $0.61(0.31-$ & 0.55 & $<$ \\
& $0.75)$ & $0.68)$ & & $0.78)$ & $0.23-$ & 0.001 \\
& & & & & $0.73)$ & \\
\hline
\end{tabular}

* EPI = echo-planar imaging, $E T L=$ echo train length, $T R=$ repetition time,$P=p$ value,$C C C=$ concordance correlation coefficients, $\mathrm{AlH}=$ acute ischemic hyperintensity, $\mathrm{ClH}=$ chronic ischemic hyperintensity

** Parentheses indicate numbers of radiomics features in proportions of stable radiomics features or indicate $95 \%$ confidence intervals.

\section{Stable radiomic features in fast scanned FLAIR compared with conventional FLAIR}

There were no stable radiomic features across acute and chronic ischemic hyperintensities in the first and second groups. Stable radiomic features across acute and chronic ischemic hyperintensities in the first group were $1.01 \%$ (1/99) in GLRLM and 2.22\% (1/45) in NGTDM. Stable radiomic features across acute and chronic ischemic hyperintensities in the second group were $1.01 \%(1 / 99)$ in GLRLM. Stable radiomic features across acute ischemic hyperintensities in the first group were $2.17 \%(1 / 46)$ in the first order, $2.53 \%(5 / 198)$ in GLCM, 4.04\% (4/99) in GLRLM, 2.22\% (1/45) in LBP, 1.71\% (2/117) in GLSZM, and $6.67 \%(3 / 45)$ in NGTDM. Stable radiomic features across chronic ischemic hyperintensities in the first group were 4.44\% (2/45) in the second order, 2.02\% (4/198) in GLCM, 6.06\% (6/99) in GLRLM, 4.27\% $(5 / 117)$ in GLSZM, and 4.44\% (2/45) in NGTDM. Stable radiomic features across acute ischemic hyperintensities in the second group were $2.17 \%(1 / 46)$ in the first order, 2.02\% (4/198) in GLCM, 2.02\% $(2 / 99)$ in GLRLM, 2.22\% (1/45) in LBP, 0.85\% (1/117) in GLSZM, and 4.44\% (2/45) in NGTDM. Stable radiomic features across chronic ischemic hyperintensities in the second group were $1.01 \%(1 / 99)$ in GLRLM, 2.22\% (1/45) in LBP, and 2.22\% (1/45) in NGTDM.

There were no stable radiomic features across acute and chronic ischemic hyperintensities using intersection ROls in the first group. Stable radiomic features across acute and chronic ischemic hyperintensities in the second group were 1.01\% (1/99) in GLRLM and 2.22\% (1/45) in LBP. Stable radiomic features across acute ischemic hyperintensities in the first group were $4.35 \%(2 / 46)$ in the first order, $2.22 \%$ (1/45) in the second order, $1.01 \%$ (2/198) in GLCM, 4.04\% (4/99) in GLRLM, 2.22\% (1/45) in 
LBP, and $1.71 \%(2 / 117)$ in GLSZM. Stable radiomic features across chronic ischemic hyperintensities in the first group were $2.22 \%(1 / 45)$ in the second order, $2.53 \%$ (5/198) in GLCM, 6.06\% (6/99) in GLRLM, $5.13 \%(6 / 117)$ in GLSZM, and 4.44\% (2/45) in NGTDM. Stable radiomic features across acute ischemic hyperintensities in the second group were 6.52\% (3/46) in the first order, 3.03\% (6/198) in GLCM, 5.05\% (5/99) in GLRLM, 2.22\% (1/45) in LBP, 2.56\% (3/117) in GLSZM, and 4.44\% (2/45) in NGTDM. Stable radiomic features across chronic ischemic hyperintensities in the second group were $1.01 \%(1 / 99)$ in GLRLM and 4.44\% (2/45) in LBP. All of the details mentioned above are shown in Figs. 1-3.

\section{Discussion}

This study showed a consistent tendency of higher proportions of reliable features in EPI-FLAIR and TRFLAIR than ETL-FLAIR in both acute and chronic ischemic hyperintensities and for both whole-and intersection-ROI mask. Therefore, various image acquisitions of FLAIR resulted in unstable radiomic features, which may lead to different radiomic features' outcomes, such as prediction modeling.

MRI is a useful and sometimes essential imaging modality to identify the infarct core on DWI, and additional useful information can be obtained from various images such as FLAIR or gradient echo (GRE) images, and also allow acquisition of vessel information without the need for contrast media during the evaluation of cerebral acute ischemic stroke patients; however, MRI has a lesser availability and a longer scan time compared to $\mathrm{CT}{ }^{5,18-20}$. Therefore, there have been many attempts to reduce the scan time of MRI in cerebral acute ischemic stroke situations, which has resulted in various MRI sequences and parameters being applied in clinical practice ${ }^{6,9-12}$.

The diversity in the image acquisition makes it difficult to apply radiomic features to MRI for cerebral acute ischemic stroke. Imaging acquisition, segmentation, and feature extraction can affect the stability of radiomic features ${ }^{2}$. Ford et al. demonstrated that changes of imaging parameters could lead to variable radiomic features in a phantom study ${ }^{21}$. In addition, these obstacles may affect a few published studies on the predictive models developed using MRI radiomic features in cerebral acute ischemic stroke 22-24. The results from this study showed some stable radiomic features across variable acquisition of FLAIRs and acute and chronic ischemic hyperintensities, but a substantial proportion showed variability. To our knowledge, there is no previous report on the stability of MRI radiomic features according to various imaging parameters in cerebral acute ischemic stroke.

Studies evaluating stable radiomic features in cerebral acute or chronic ischemic lesions based on multiparameteric variances appear to be lacking, and the reproducibility of radiomic features in brain tumors, including glioblastoma, has been reported and several features belonging to GLRLM were identified as reproducible features ${ }^{25,26}$. GLRLM was also the most reproducible feature in cine balanced steady-state free procession and first-order, and GLCM had the most reproducible features on both T1 and T2 maps in the myocardial radiomic features ${ }^{27}$. However, a phantom study for test-retest reproducibility reported that GLRLM was neither the most robust nor least robust feature class, while GLCM was one of the least robust feature classes across MRI sequences: FLAIR, T1-weighted, and T2-weighted imaging 28 . 
Our study also showed that GLCM and GLRLM are common stable features in the numbers but some variability was seen in the proportions of stable radiomic features.

This study has several limitations. First, this study was designed as a retrospective study with a small population. Second, we did not compare all of the FLAIRs simultaneously because it is hard to obtain all of the FLAIRs at the same time in a cerebral acute ischemic stroke situation. Third, this study presented only the stabilities of radiomic features in FLAIRs of acute ischemic stroke and an evaluation of the stability of identification or prediction models for stroke outcomes using radiomic features from variable FLAIRs is necessary.

In conclusion, the fast-scanned FLAIR showed small proportions of stable radiomic features and variable stability of radiomic features among those in terms of the agreements with conventional FLAIR. Therefore, even if the images in the same sequence have different parameters, the radiomic features obtained from the images may be used with caution.

\section{Methods}

\section{Study population}

From May 2015 to June 2016, 296 patients undergoing MRI at a single tertiary hospital for symptoms of acute ischemic stroke were retrospectively enrolled. Among them, 118 patients underwent echo-planar imaging (EPI)-FLAIR and echo train length (ETL)-FLAIR and 178 patients underwent ETL-FLAIR and repetition time (TR)-FLAIR simultaneously. Seventy-nine patients showed acute ischemic hyperintensity and 89 patients showed chronic ischemic hyperintensity on simultaneous acquisition of EPI- and ETLFLAIR, who were allocated to the first group, and 112 patients showed acute ischemic hyperintensity and 127 patients showed chronic ischemic hyperintensity on simultaneous acquisition of ETL- and TR-FLAIR, who were allocated to the second group. The detailed demographics of the patients are presented in Table 2. The enrolled patients were collected from the fast stroke MRI registry in our institute ${ }^{6}$. The institutional review board of Asan Medical Center approved the present study, and the requirement for informed consent was waived. The data was analyzed in compliance with the International Council for Harmonization of Technical Requirements for Registration of Pharmaceutical for Human Use: Guideline for Good Clinical Practice (ICH GCP).

\section{Table 2. Demographics of the patients}




\begin{tabular}{|c|c|c|c|c|c|c|}
\hline & \multicolumn{3}{|c|}{ Acute ischemic lesion } & \multicolumn{3}{|c|}{$\begin{array}{l}\text { Chronic ischemic } \\
\text { lesion }\end{array}$} \\
\hline & $\begin{array}{l}\text { EPI-ETL } \\
(n=79)\end{array}$ & $\begin{array}{l}\text { ETL-TR } \\
(n=112)\end{array}$ & $\begin{array}{l}P \\
\text { value }\end{array}$ & $\begin{array}{l}\text { EPI-ETL } \\
(n=89)\end{array}$ & $\begin{array}{l}\text { ETL-TR } \\
(n=127)\end{array}$ & $\begin{array}{l}P \\
\text { value }\end{array}$ \\
\hline Age (years) ${ }^{a}$ & $\begin{array}{l}67.9 \pm \\
13.2 \\
92)\end{array}$ & $\begin{array}{l}67.8 \pm 13.4 \\
(22-95)\end{array}$ & 0.95 & $\begin{array}{l}69.4 \pm \\
10.9 \\
(38-92)\end{array}$ & $\begin{array}{l}72.4 \pm \\
9.4 \\
(38-95)\end{array}$ & 0.01 \\
\hline $\operatorname{Sex}(M: F)$ & $46: 33$ & $73: 39$ & 0.41 & $52: 37$ & $74: 53$ & 0.91 \\
\hline $\begin{array}{l}\text { Body mass } \\
\text { index }\left(\mathrm{kg} / \mathrm{m}^{2}\right)^{a}\end{array}$ & $\begin{array}{l}23.9 \pm 3.5 \\
(16.4-32.1)\end{array}$ & $\begin{array}{l}24.1 \pm 3.4 \\
(13.8-36.6)\end{array}$ & 0.76 & $\begin{array}{l}23.3 \pm \\
2.9 \\
(15.1- \\
32.1)\end{array}$ & $\begin{array}{l}23.4 \pm \\
3.4 \\
(13.8- \\
36.6)\end{array}$ & 0.86 \\
\hline Hypertension ${ }^{b}$ & $54(68.4)$ & $70(63.1)$ & 0.5 & $64(71.9)$ & $92(73.0)$ & 0.95 \\
\hline $\mathrm{DM}^{\mathrm{b}}$ & $28(35.4)$ & $29(26.1)$ & 0.21 & $33(37.1)$ & $43(34.1)$ & 0.73 \\
\hline Hyperlipidemia $^{\text {b }}$ & $34(43.0)$ & 37 (33.6) & 0.21 & 35 (39.3) & $43(34.7)$ & 0.5 \\
\hline $\begin{array}{l}\text { Smoking history } \\
b \mathrm{~b}\end{array}$ & $32(40.5)$ & $43(38.7)$ & 0.89 & $36(40.4)$ & $84(33.3)$ & $<0.01$ \\
\hline $\begin{array}{l}\text { History of stroke } \\
\mathrm{b}\end{array}$ & $17(21.5)$ & $24(21.6)$ & $<0.01$ & $25(28.1)$ & $46(37.1)$ & 0.27 \\
\hline $\begin{array}{l}\text { Family history } \\
\text { of stroke }\end{array}$ & $19(24.1)$ & $33(29.7)$ & 0.51 & $20(22.5)$ & $30(24)$ & 0.97 \\
\hline $\begin{array}{l}\text { History of } \\
\text { coronary } \\
\text { disease }^{b}\end{array}$ & $26(32.9)$ & $42(37.8)$ & 0.62 & $30(33.7)$ & $45(36)$ & 0.91 \\
\hline $\begin{array}{l}\text { Onset to } \\
\text { imaging time }\end{array}$ & $\begin{array}{l}258 \\
683)^{\mathrm{c}}\end{array}$ & $\begin{array}{l}223 \\
700)^{c}\end{array}(123-$ & 0.65 & $\begin{array}{l}261 \\
(123.5- \\
576)^{c}\end{array}$ & $\begin{array}{l}172 \\
(117.5- \\
306.5)^{\mathrm{c}}\end{array}$ & 0.10 \\
\hline
\end{tabular}

a Numbers indicate mean \pm standard deviation and the parentheses indicate the range.

${ }^{b}$ Parentheses indicate the range.

${ }^{\mathrm{c}}$ Median (nterquartile range) 
All FLAIR was scanned on a 1.5-T scanner (Magnetom Avanto; Siemens Healthineers). The scan times were 128 seconds for conventional FLAIR, 45 seconds for EPI-FLAIR, 74 seconds for ETL-FLAIR, and 79 seconds for TR-FLAIR. The detailed scan parameters for the conventional and fast FLAIR were as previously reported ${ }^{6}$ and are listed in Table 3 and representative images are presented in Figure 4.

\section{Table 3. FLAIR protocols}

\begin{tabular}{|lllll|}
\hline & FLAIR & & & \\
\hline & Conventional & EPI & ETL & TR \\
\hline TR/TE & $9000 / 109$ & $9000 / 101$ & $9000 / 102$ & $5560 / 109$ \\
\hline Inversion time & 2500 & 2000 & 2500 & 1930 \\
\hline Flip angle & 150 & 180 & 150 & 150 \\
\hline Matrix & $256 \times 218$ & $128 \times 128$ & $192 \times 192$ & $256 \times 256$ \\
\hline Field of view & $210 \times 184$ & $230 \times 230$ & $210 \times 184$ & $210 \times 210$ \\
\hline Number of slices & 20 & 20 & 20 & 20 \\
\hline Slice thickness (mm) & 5 & 5 & 5 & 5 \\
\hline Slice gap (mm) & 2 & 2 & 2 & 2 \\
\hline ETL & 21 & $128($ EPI) & 32 & 21 \\
\hline Acceleration factor & 2 & 2 & 2 & 2 \\
\hline NEX & 1 & 2 & 1 & 1 \\
\hline Scan time & 128 & 45 & 74 & 79 \\
\hline
\end{tabular}

* EPI= echo-planar imaging, ETL= echo train length, $T R=$ repetition time, $T E=$ echo time, NEX= number of excitations

Image analysis

The segmentations of acute- and chronic ischemic hyperintensities were conducted as described in a previous report ${ }^{6}$. We defined acute ischemic hyperintensity as a FLAIR high signal intensity within acute infarcts on diffusion weighted images (DWI) ${ }^{15,17}$, and chronic ischemic hyperintensity as a hyperintensity outside of acute infarcts on DWI. The segmentation of region-of-interest (ROI) mask was done by one research assistant (K.M.C. with 5 years of experience) using an in-house program for semiautomatic segmentation (based on ImageJ software; National Institutes of Health, Bethesda, MD). The intersection 
ROI mask between the respective ROI mask of the conventional and fast FLAIR was obtained after the coregistration and then each intersection ROI mask was transferred into the respective conventional and fast FLAIR images. A total of 14 kinds of ROI masks were obtained as follows: 3 ROls from conventional-, EPI-, and ETL-FLAIR in the first group; 2 intersection ROIs from conventional and EPI-FLAIR, and 2 intersection ROIs from conventional and ETL-FLAIR in the first group; 3 ROIs from conventional-, ETL-, and TR-FLAIR in the second group; 2 intersection ROIs from conventional and ETL-FLAIR, and 2 intersection ROIs from conventional and TR-FLAIR in the second group (Figure 5). From the ROIs, 595 radiomics features were extracted and concordance correlation coefficients (CCC) for radiomic features were calculated between fast scanned and conventional FLAIR in each group.

Radiomic features

Radiomic features were extracted with Matlab R2016a (The Mathworks, Natick, MA): first-order features, texture features, and wavelet-transformed features ${ }^{29,30}$. The first-order features were acquired based on the histogram analyses of pixel values within the region-of-interest. The second-order features or texture features were as follows: gray-level co-occurrence matrix (GLCM), gray-level run-length matrix (GLRLM), local binary pattern (LBP), gray-level size zone matric (GLSZM), and neighboring gray tone difference matrix (NGTDM). The wavelet transformations extracted additional features. The radiomic features extraction was done under the Imaging Biomarker Standardization Initiative ${ }^{31}$. Finally, 595 radiomic features were extracted with 46 first-order features, 61 texture features ( 5 second order, 22 GLCM, 11 GLRLM, 5 LBP, 13 GLSZM, 5 NGTDM) and 488 wavelet features (x8; 40 second order, 176 GLCM, 88 GLRLM, 40 LBP, 104 GLSZM, 40 NGTDM).

Statistical analysis

The stability of the radiomic features was evaluated using CCC between the features extracted from the conventional- and fast scanned FLAIR, followed Lin's definition ${ }^{32}$. The proportions of stable radiomic features were compared between EPI and ETL in the first group and between TR and ETL in the second group using McNemar's test. Radiomic features with a CCC of higher than 0.85 were considered to be stable. All statistical analyses were performed using the MedCalc 15.6.1 software package (MedCalc Software) and R version R 3.3.3 (R Foundation for Statistical Computing; http://www.R-project.org, 2016).

\section{Declarations}

\section{Acknowledgements}

Figures 1 and 2 were drawn by L. J., Figure 3 was drawn by S. Y. P using R version R 3.3.3 (R Foundation for Statistical Computing; http://www.R-project.org, 2016), and Figures 4 ands 5 were drawn by S.C.J.

\section{Author Contributions}

L. J. made contributions to the analysis/interpretation of data and made the first draft of this work. 
S. C. J. is responsible for the design and conception of this work, supervised the entire process and completed the revision of the first draft.

H, L. made contributions to feature extraction and analysis, and completed the revision of the first draft.

S. Y. P. took responsibility for the statistical analysis and advice.

M. K. made contributions to the analysis/interpretation of the data.

J. E. P. made contribution to this work with advice in terms of radiomics.

K. M. C. contributed to this work by data acquisition.

\section{Additional Information}

The author(s) declare no competing interests.

\section{References}

1 Gillies, R. J., Kinahan, P. E. \& Hricak, H. Radiomics: Images Are More than Pictures, They Are Data. Radiology278, 563-577, doi:10.1148/radiol.2015151169 (2016).

2 Park, J. E., Park, S. Y., Kim, H. J. \& Kim, H. S. Reproducibility and Generalizability in Radiomics Modeling: Possible Strategies in Radiologic and Statistical Perspectives. Korean J Radio/20, 1124-1137, doi:10.3348/kjr.2018.0070 (2019).

3 van Timmeren, J. E., Cester, D., Tanadini-Lang, S., Alkadhi, H. \& Baessler, B. Radiomics in medical imaging-"how-to" guide and critical reflection. Insights Imaging11, 91, doi:10.1186/s13244-020-00887-2 (2020).

4 Zwanenburg, A. et al. The Image Biomarker Standardization Initiative: Standardized Quantitative Radiomics for High-Throughput Image-based Phenotyping. Radiology295, 328-338, doi:10.1148/radiol.2020191145 (2020).

5 Kohrmann, M. \& Schellinger, P. D. Acute stroke triage to intravenous thrombolysis and other therapies with advanced CT or MR imaging: pro MR imaging. Radiology251, 627-633, doi:10.1148/radiol.2513081074 (2009).

6 Chung, M. S. et al. Reliability of fast magnetic resonance imaging for acute ischemic stroke patients using a 1.5-T scanner. Eur Radio/29, 2641-2650, doi:10.1007/s00330-018-5812-5 (2019).

$7 \quad \mathrm{Li}, \mathrm{B}$., Li, H., Dong, L. \& Huang, G. Fast carotid artery MR angiography with compressed sensing based three-dimensional time-of-flight sequence. Magn Reson Imaging43, 129-135, doi:10.1016/j.mri.2017.07.017 (2017). 
8 Li, B. et al. Compressed sensing based simultaneous black- and gray-blood carotid vessel wall MR imaging. Magn Reson Imaging38, 214-223, doi:10.1016/j.mri.2017.01.013 (2017).

9 Meshksar, A. et al. Role of EPI-FLAIR in patients with acute stroke: a comparative analysis with FLAIR. AJNR Am J Neuroradio/35, 878-883, doi:10.3174/ajnr.A3786 (2014).

10 Nael, K. et al. Six-minute magnetic resonance imaging protocol for evaluation of acute ischemic stroke: pushing the boundaries. Stroke45, 1985-1991, doi:10.1161/STROKEAHA.114.005305 (2014).

11 Lin, Z., Guo, Z., Qiu, L., Yang, W. \& Lin, M. The applied research of MRI with ASSET-EPI-FLAIR combined with 3D TOF MRA sequences in the assessment of patients with acute cerebral infarction. Acta Radio/57, 1515-1523, doi:10.1177/0284185116628338 (2016).

$12 \mathrm{JM}, \mathrm{U}$. K.-I. et al. Utility of an ultrafast magnetic resonance imaging protocol in recent and semirecent strokes. J Neurol Neurosurg Psychiatry76, 1002-1005, doi:10.1136/jnnp.2004.046201 (2005).

13 Feinberg, D. A. \& Setsompop, K. Ultra-fast MRI of the human brain with simultaneous multi-slice imaging. J Magn Reson229, 90-100, doi:10.1016/j.jmr.2013.02.002 (2013).

14 Song, S. S. et al. A pragmatic approach using magnetic resonance imaging to treat ischemic strokes of unknown onset time in a thrombolytic trial. Stroke43, 2331-2335, doi:10.1161/STROKEAHA.111.630947 (2012).

15 Ebinger, M. et al. Fluid-attenuated inversion recovery evolution within 12 hours from stroke onset: a reliable tissue clock? Stroke41, 250-255, doi:10.1161/STROKEAHA.109.568410 (2010).

16 Lee, K. Y. et al. Distal hyperintense vessels on FLAIR: an MRI marker for collateral circulation in acute stroke? Neurology72, 1134-1139, doi:10.1212/01.wnl.0000345360.80382.69 (2009).

17 Petkova, M. et al. MR imaging helps predict time from symptom onset in patients with acute stroke: implications for patients with unknown onset time. Radiology257, 782-792, doi:10.1148/radiol.10100461 (2010).

18 Vert, C., Parra-Farinas, C. \& Rovira, A. MR imaging in hyperacute ischemic stroke. European journal of radiology $96,125-132$, doi:10.1016/j.ejrad.2017.06.013 (2017).

19 Rudkin, S., Cerejo, R., Tayal, A. \& Goldberg, M. F. Imaging of acute ischemic stroke. Emergency radiology25, 659-672, doi:10.1007/s10140-018-1623-x (2018).

20 Muir, K. W., Buchan, A., von Kummer, R., Rother, J. \& Baron, J.-C. Imaging of acute stroke. The Lancet Neurology5, 755-768, doi:10.1016/s1474-4422(06)70545-2 (2006).

21 Ford, J., Dogan, N., Young, L. \& Yang, F. Quantitative Radiomics: Impact of Pulse Sequence Parameter Selection on MRI-Based Textural Features of the Brain. Contrast Media Mol Imaging2018, 
1729071, doi:10.1155/2018/1729071 (2018).

22 Cui, H., Wang, X., Bian, Y., Song, S. \& Feng, D. D. Ischemic stroke clinical outcome prediction based on image signature selection from multimodality data. Annu Int Conf IEEE Eng Med Biol Soc2018, 722725, doi:10.1109/EMBC.2018.8512291 (2018).

23 Tang, T. Y. et al. Penumbra-based radiomics signature as prognostic biomarkers for thrombolysis of acute ischemic stroke patients: a multicenter cohort study. J Neuro/267, 1454-1463, doi:10.1007/s00415-020-09713-7 (2020).

24 Zhang, R. et al. Apparent diffusion coefficient map based radiomics model in identifying the ischemic penumbra in acute ischemic stroke. Ann Palliat Med9, 2684-2692, doi:10.21037/apm-20-1142 (2020).

25 Li, Q. et al. A Fully-Automatic Multiparametric Radiomics Model: Towards Reproducible and Prognostic Imaging Signature for Prediction of Overall Survival in Glioblastoma Multiforme. Sci Rep7, 14331, doi:10.1038/s41598-017-14753-7 (2017).

26 Shiri, I. et al. Repeatability of radiomic features in magnetic resonance imaging of glioblastoma: Test-retest and image registration analyses. Med Phys47, 4265-4280, doi:10.1002/mp.14368 (2020).

27 Jang, J. et al. Reproducibility of Segmentation-based Myocardial Radiomic Features with Cardiac MRI. Radiol Cardiothorac Imaging2, e190216, doi:10.1148/ryct.2020190216 (2020).

28 Baessler, B., Weiss, K. \& Pinto Dos Santos, D. Robustness and Reproducibility of Radiomics in Magnetic Resonance Imaging: A Phantom Study. Invest Radio/54, 221-228, doi:10.1097/RLI.0000000000000530 (2019).

29 Kickingereder, P. et al. Radiomic Profiling of Glioblastoma: Identifying an Imaging Predictor of Patient Survival with Improved Performance over Established Clinical and Radiologic Risk Models. Radiology280, 880-889, doi:10.1148/radiol.2016160845 (2016).

30 Kang, D. et al. Diffusion radiomics as a diagnostic model for atypical manifestation of primary central nervous system lymphoma: development and multicenter external validation. Neuro Oncol, doi:10.1093/neuonc/noy021 (2018).

31 Zwanenburg, A., Leger, S., Vallières, M. \& Löck, S. Image biomarker standardisation initiative. arXiv preprint arXiv:1612.07003(2016).

32 Lin, L. I. A Concordance Correlation-Coefficient to Evaluate Reproducibility. Biometrics45, 255-268, doi:Doi 10.2307/2532051 (1989).

\section{Figures}



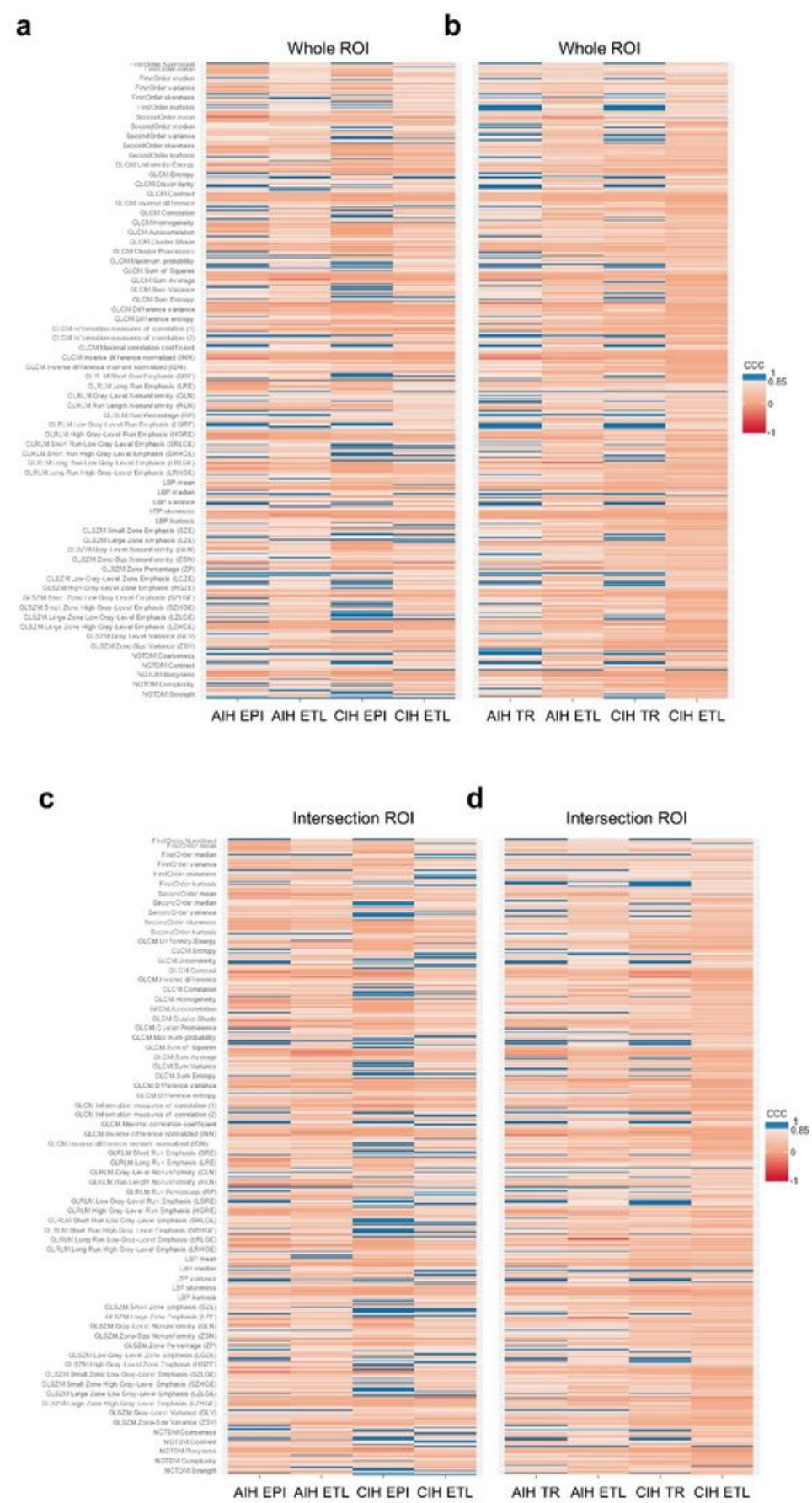

\section{Figure 1}

Heat maps of radiomics features extracted from whole- and intersection ROI masks in the first and second groups. Features with CCC $>0.85$ were regarded as stable. (a) Features from whole ROI masks in the first group. (b) Features from whole ROI masks in the second group. (c) Features from intersection ROI masks in the first group. (d) Features from intersection ROI masks in the second group. AlH, acute 
ischemic hyperintensity; $\mathrm{ClH}$, chronic ischemic hyperintensity; $\mathrm{CCC}$, concordance correlation coefficients; ROI, region-of-interest; EPI, echo-planar imaging; ETL, echo train length; TR, repetition time.

a

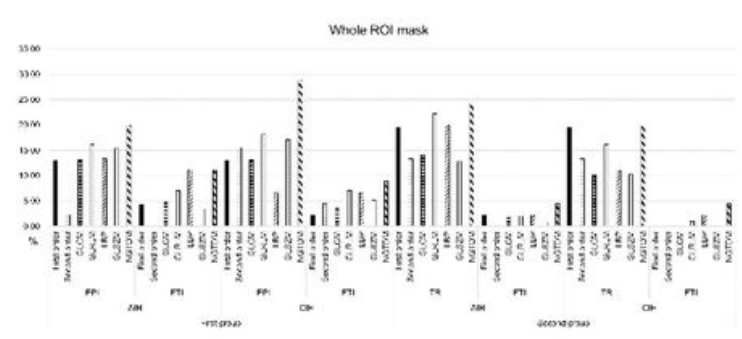

C

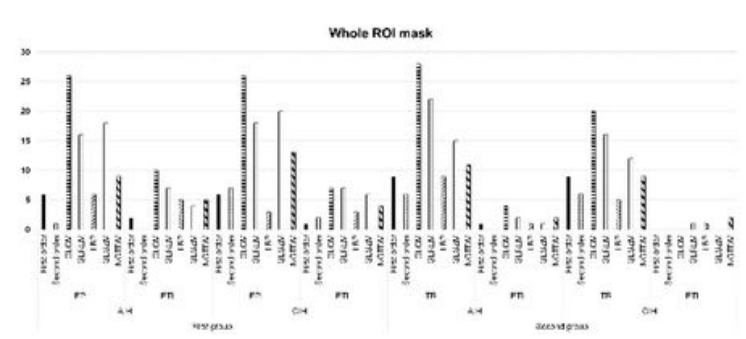

b

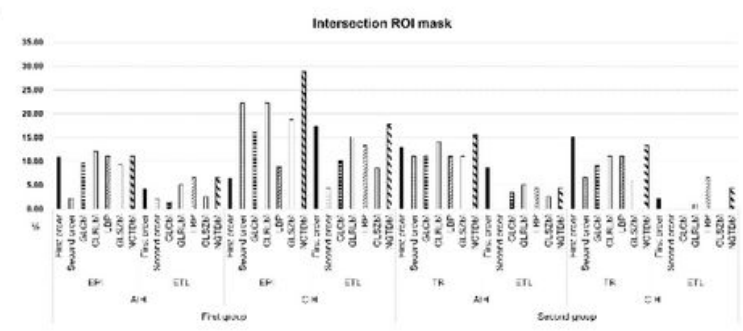

d

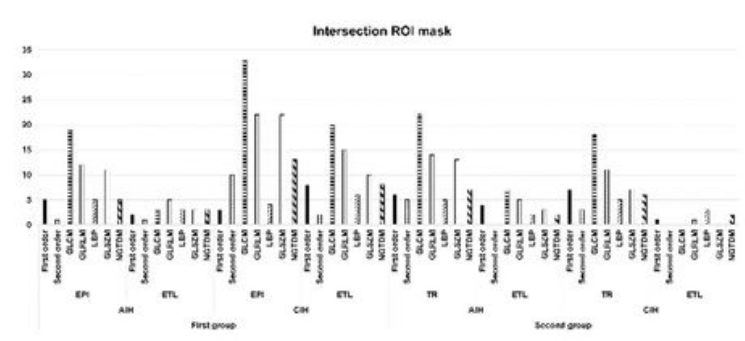

\section{Figure 2}

Proportions of stable radiomic features extracted from whole- and intersection ROI masks on each fast scanned FLAIR sequence in the first and second groups. $(a, b)$ Proportions of stable radiomic features from whole ROI masks (a) and intersection ROI masks (b) according to each fast scanned FLAIR. (c, d) Numbers of stable radiomic features from whole ROI masks (c) and intersection ROI masks (d) according to each fast scanned FLAIR. AlH, acute ischemic hyperintensity; $\mathrm{ClH}$, chronic ischemic hyperintensity; ROI, region-of-interest; EPI, echo-planar imaging; ETL, echo train length; TR, repetition time; gray-level cooccurrence matrix, GLCM; gray-level run-length matrix, GLRLM; local binary pattern, LBP; gray-level size zone matric, GLSZM; neighboring gray tone difference matrix, NGTDM 

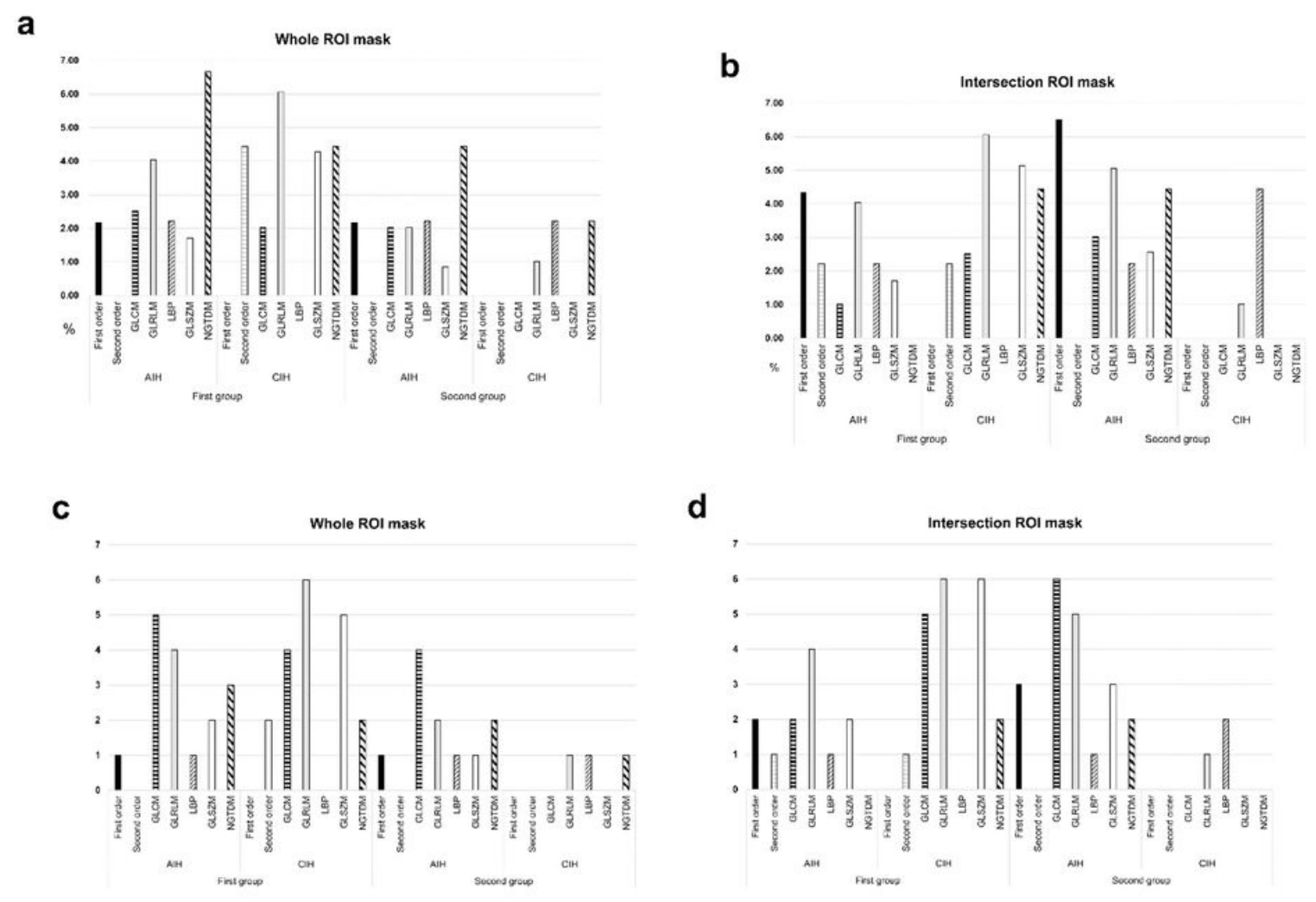

Figure 3

Proportions of stable radiomic features throughout both fast scanned FLAIR sequences extracted from whole- and intersection ROI masks in the first and second groups. (a, b) Proportions of stable radiomic features from whole ROI masks (a) and intersection ROI masks (b). (c, d) Numbers of stable radiomic features from whole ROI masks (c) and intersection ROI masks (d). AlH, acute ischemic hyperintensity; $\mathrm{CIH}$, chronic ischemic hyperintensity; ROI, region-of-interest; EPI, echo-planar imaging; ETL, echo train length; TR, repetition time; gray-level co-occurrence matrix, GLCM; gray-level run-length matrix, GLRLM; local binary pattern, LBP; gray-level size zone matric, GLSZM; neighboring gray tone difference matrix, NGTDM 


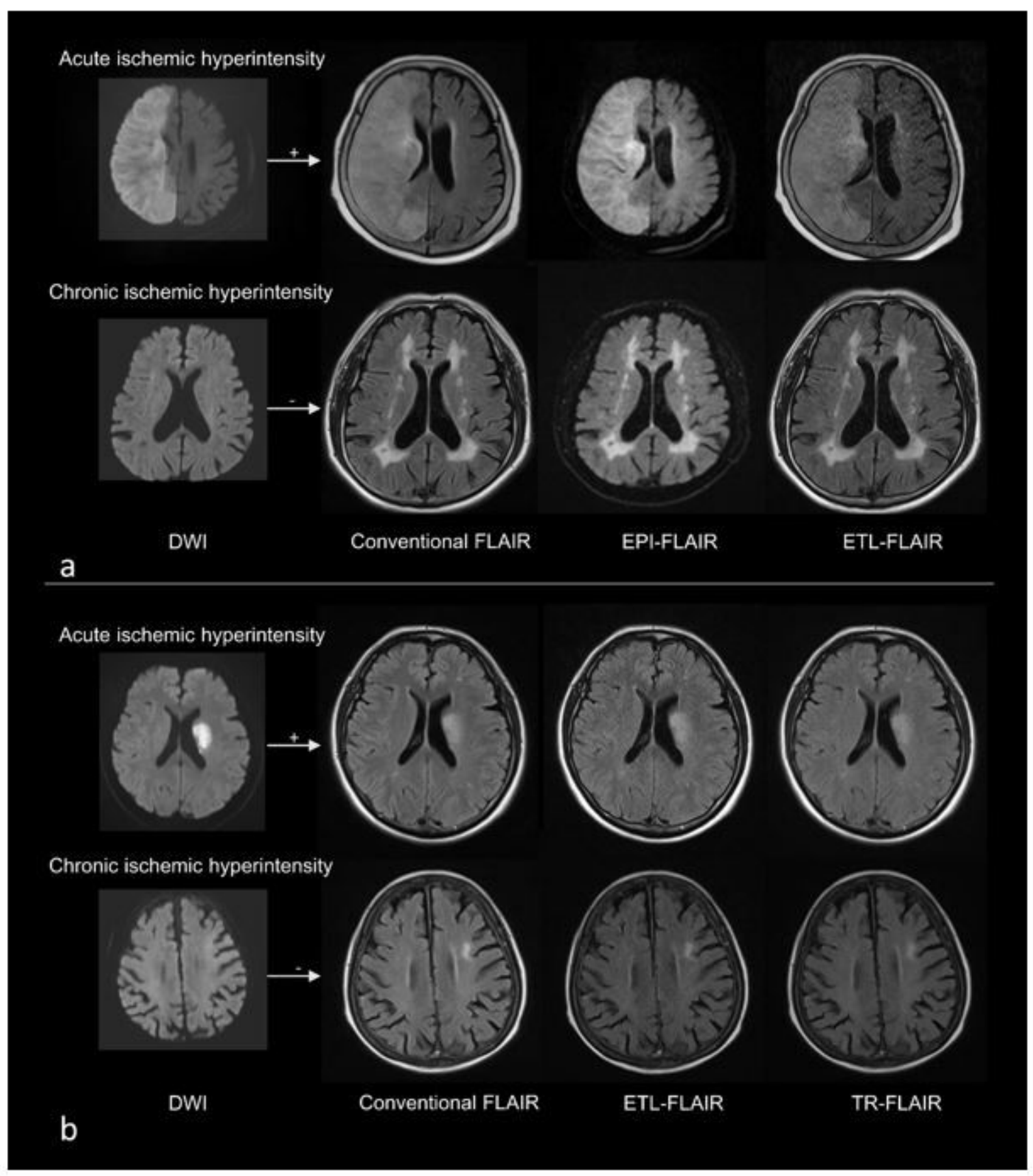

\section{Figure 4}

Acute and chronic ischemic hyperintensities on conventional FLAIR and fast scanned FLAIR with respect to DWI. (a) First group with conventional FLAIR, EPI-FLAIR, and ETL-FLAIR. (b) Second group with conventional FLAIR, ETL-FLAIR and TR-FLAIR. DWI, diffusion-weighted image; FLAIR, fluid attenuated inversion recovery; $\mathrm{EPI}$, echo-planar imaging; ETL, echo train length; TR, repetition time 


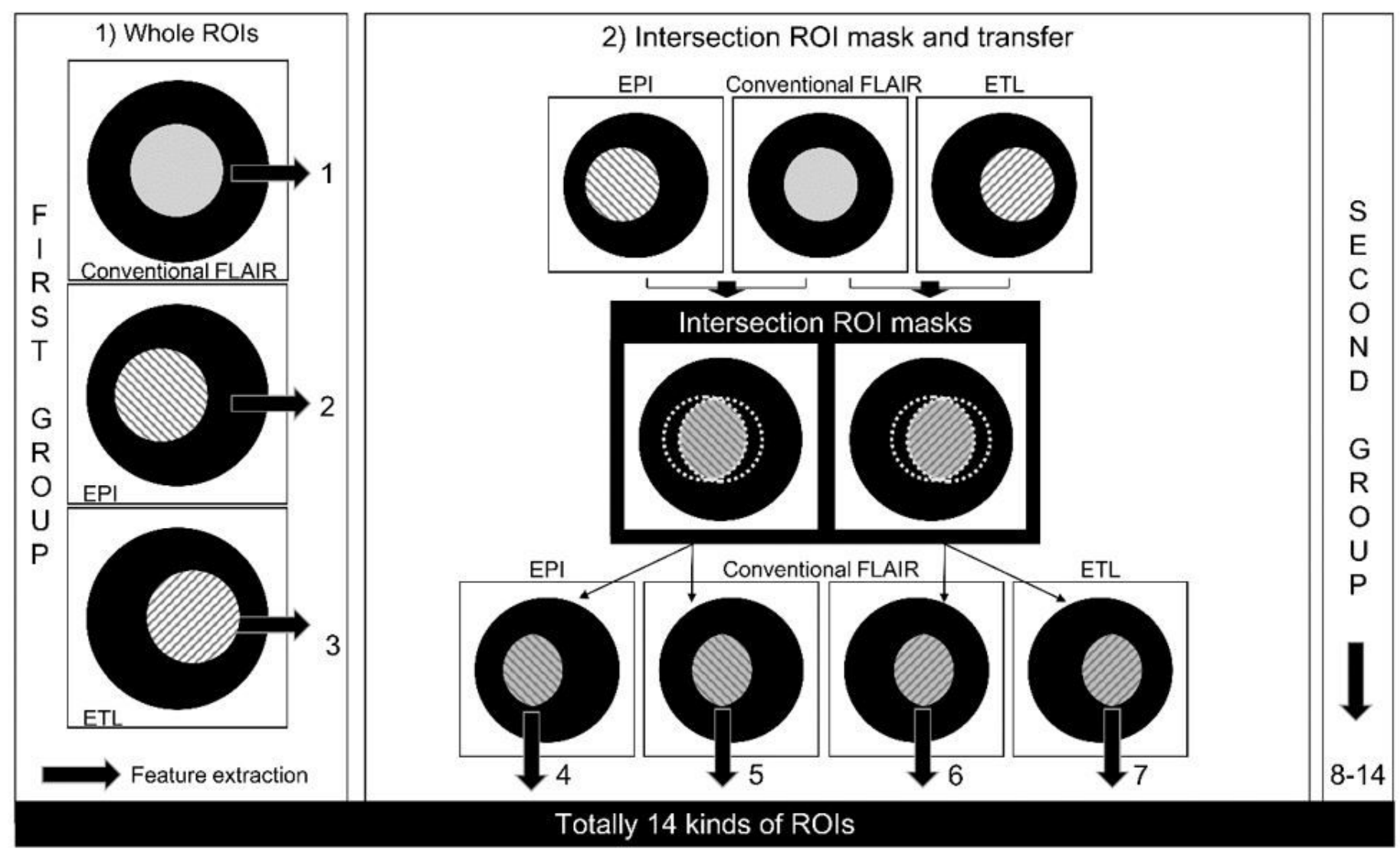

Figure 5

The outline of the image analysis focused on the types of ROI masks used in this study. Feature extraction was performed from each conventional and fast scanned FLAIR using both whole-( $(x 3)$ and intersection ROI masks ( $x 4$ ) for both the first and second groups ( $x 2$ ). FLAIR, fluid attenuated inversion recovery; ROI, region-of-interest; EPI, echo-planar imaging; ETL, echo train length; $T R$, repetition time. 